\title{
Is It Time to Monitor Flow Bias During Mechanical Ventilation?
}

During invasive mechanical ventilation, the inspiratory and expiratory air flows are correlated and dependent on the ventilator settings and patient characteristics such as tidal volume and pulmonary compliance. Inspiratory flow, however, is easier to manipulate than expiratory flow during controlled mechanical ventilation. While inspiratory flow depends mainly on tidal volume, inspiratory time, and the selected flow pattern, expiratory flow is determined not only by PEEP and tidal volume, but also (and fundamentally) by airway resistance and pulmonary compliance. The mechanical properties of the respiratory system can exert some influence on inspiratory peak flow only during pressure controlled or pressure support modes.

Independent of the generated air flows, the net volume of gas that moves into and out of the lungs during most normal respiratory cycles tends to be equal, on average. Otherwise we would observe lung inflation or deflation over the course of a few respiratory cycles. The same is not true, however, for the peak or mean flow during the inspiratory and expiratory phases, which can differ substantially, resulting in flow bias. A higher peak inspiratory flow (PIF) than peak expiratory flow (PEF) generates a flow bias toward the lungs, and the inverse (ie, PIF $<$ PEF) generates a flow bias toward the mouth.

Since the 1970s, and especially in the past few years, there has been a growing, although still incipient, concern about the potential influence of flow bias on mucus movement. According to experimental studies, a PIF-PEF difference higher than $17 \mathrm{~L} / \mathrm{min}$ or a PIF/PEF ratio higher than 1.1 (thresholds that should be interpreted with caution) may move secretions deeper into the lungs and may also increase the risk of inflammation propagation by the movement of biological fluids between 2 lung regions with different compliances. ${ }^{1-3}$

The ventilation mode, tidal volume, and inspiratory time are usually the first variables chosen when starting mechanical ventilation, and because the adjustment of the latter 2 are pre-established upon certain clinical conditions, inspiratory flow is often a consequence of those choices. In this issue of Respiratory CARE, Ntoumenopoulos et al present a preliminary study that found that common ventilator settings generate an inspiratory flow bias. ${ }^{4}$ Nineteen of the 20 patients they studied had a PIF/PEF ratio higher than 1.1 , and 8 of the 20 patients an absolute mean inspiratory flow bias greater than $17 \mathrm{~L} / \mathrm{min}$. None of the patients had an expiratory flow bias, which, theoretically, would favor mucus clearance.

See the Original Study on Page 1887

The study by Ntoumenopoulos et $\mathrm{al}^{4}$ confirms a previous suspicion that in clinical practice an inspiratory flow bias is often imposed by mechanical ventilation. ${ }^{5}$ That finding is concerning and evinces the urgent need for more studies, especially in animals, to investigate the actual influence of flow bias on mucus movement. If certain ventilation settings substantially hinder secretion clearance in large animals, we should quickly move to quantify this potentially harmful phenomenon in humans.

An inspiratory flow bias might be beneficial in certain clinical conditions such as the initial phase of ARDS. For instance, we could hypothesize that the use of a low tidal volume in that condition might be beneficial, not only because it avoids lung injury, but also because it avoids generating high elastic recoil pressure at end inspiration, thus reducing expiratory peak flow. The inspiratory flow bias thus generated (low PEF and normal-range PIF) might help contain the spread of inflammatory mediators. ${ }^{5,6}$ Conversely, a high PIF (commonly applied in patients with COPD, to shorten the inspiratory phase and prolong the expiratory phase) might also generate an inspiratory flow bias that would be enhanced by flow limitation during exhalation, but in this case it might contribute to mucus retention, a potentially hazardous consequence.

Another potential area of application of these concepts is chest physiotherapy. Secretion-clearance techniques might be optimized by including a temporary expiratory flow bias. During the therapy, or a few minutes before starting it, the inspiratory flow might be reduced and the tidal volume slightly increased to create an expiratory flow bias. Paralyzed patients and patients with ineffective cough or mucus hypersecretion would be the most likely to benefit from this pre-facilitation of mucus clearance.

Future research about secretion movement in mechanically ventilated patients should also study the influence of gravity, and therefore body position, on mucus movement. The lung has evolved as a compartmentalized structure, 


\section{Is It Time to Monitor Flow Bias During Mechanical Ventilation?}

well designed to confine damaging biofluids to their sites of origin. 5 This compartmentalization makes the whole system very complex, subject to influences that cannot be predicted by simple physical models. It has been proposed that gravity-more than tidal air flow-might be the most important factor influencing mucus movement. ${ }^{5}$ Position and anatomy should always be considered. Other factors that may also play a role include changes in the function of mucociliary transport, thickened secretions from inadequate humidification, bed immobility, and muscle weakness. Such considerations highlight the necessity of animal studies to investigate airway secretion behavior in a biologically branched network.

Given the evidence and its possible implications, it is time to determine if we should include a quick estimate of the impact of the ventilator settings on mucus movement in our daily routine for mechanically ventilated patients.

The authors have disclosed no conflicts of interest.

Correspondence: Marcia Souza Volpe, Departamento de Fisioterapia Aplicada, Universidade Federal do Triângulo Mineiro, Av. Getulio Guaritá, 159 Uberaba, Minas Gerais, 38025-440 Brazil. E-mail: marciasvolpe@gmail.com.

DOI: $10.4187 /$ respcare. 01643
Marcia Souza Volpe PT

Applied Physiotherapy Department Federal University of Triângulo Mineiro

Minas Gerais, Brazil

Marcelo Britto Passos Amato MD

Pulmonary Department

Hospital das Clínicas

University of São Paulo

São Paulo, Brazil

\section{REFERENCES}

1. Kim CS, Sankaran S, Eldridge MA. Mucus transport by two-phase gas-liquid flow mechanism: continuous flow model. J Appl Physiol 1986;60(3):908-1017.

2. Benjamin RG, Kim CS, Chapman GA, Sackner MA. Removal of bronchial secretions by two-phase gas-liquid transport. Chest 1989; 95(3):658-663.

3. Volpe MS, Adams AB, Amato MBP, Marini JJ. Ventilatory patterns influence airway secretion movement. Respir Care 2008;53(10): 12871294.

4. Ntoumenopoulos G, Shannon H, Main E. Do commonly used ventilator settings for mechanically ventilated adults have the potential to embed secretions or promote clearance? Respir Care 2011;56(12): 1887-1892.

5. Marini JJ. Can we prevent the spread of focal lung inflammation? Crit Care Med 2010;38(Suppl):S574-S581.

6. Zanella A, Bellani G, Pesenti A. Airway pressure and flow monitoring. Curr Opin Crit Care 2010;16(3):255-260. 